\title{
Fostering a Culture of Medical Technology Innovation
}

\author{
Neel Sharma
}

Published online: 12 March 2015

(C) International Association of Medical Science Educators 2015

\begin{abstract}
Teaching on medical technology innovation is not common practice for trainees. This communication piece highlights the existence of a Biodesign class aimed to overcome this.
\end{abstract}

Keywords Biodesign · Innovation

The traditional view of medical training relies on an interplay of basic science and clinical facts with subsequent application to real-life patients. Some trainees may undertake scientific- or clinical-based research in order to enhance pathophysiology as well as current therapeutic approaches. A better understanding of health-care management and leadership as well as the academic discipline of medical education is also recognised. However, an emphasis on training in medical technology innovation is not so clear cut. When we look at the practice of medicine, technology in general plays a significant part. Examples include the pulse oximeter to assess a patient's oxygen saturation and a cannula/ vacutainer to sample blood for investigation purposes. Doctors often overlook the importance of these devices, yet without these innovations, patient care would be dramatically hindered [1]. During a recent TEDMED talk, Dr Paul Yock highlighted the importance of ensuring that technology for patients must not only enhance patient care but should be cost-effective, emphasising a need for technologies that 'cure the system' [2].

\footnotetext{
N. Sharma $(\bowtie)$

National University Hospital, Singapore, Singapore

e-mail: drneelsharma@outlook.com
}

The Singapore-Stanford Biodesign programme is an innovation in the field of medical education aiming to enhance the importance of medical technology innovation training among doctors. Medical doctors are grouped with engineers and business professionals and assigned a particular clinical need. The class size encompasses 40 participants with five faculty members. Each group tries to identify a gap in the market and develop a device that can enhance patient outcome for that particular disease state. Searching the literature ensures an understanding of the particular disease state with team brainstorming allowing for potential solutions to be designed.

The class runs for one semester and leads participants through a formal curriculum addressing the aspects of clinical need and concept selection, patent searching, prototyping, regulation and reimbursement, business modelling, sales and marketing. Teaching is didactic or small group tutorial based with assessment comprising written reports and presentations including demonstration of a final prototype.

As clinicians, we are not experts in engineering or business modelling and this interplay allows for concepts specific to a clinical need to be redefined. The process of redefining is certainly an important skill to learn particularly in an environment where clinical medicine is very much guideline driven.

\section{References}

1. Bhargava B. Welcome to BMJ Innovations. BMJ Innovations. 2015;1(1):1-2. January 1, 2015.

2. Stanford Medicine's TEDMED Innovation Reception 2014. Available from: http://www.youtube.com/watch?v=vwBu27nodVs. 